\title{
ARTICLE
}

\section{Comparison of the FLUKA, MCNPX, and PHITS Codes in Yield Calculation of Secondary Particles Produced by Intermediate Energy Proton Beam}

\author{
Joohee $\mathrm{OH}^{1}$, Hee-Seock LEE ${ }^{2 *}$, Suyel PARK ${ }^{1}$, Minho $\mathrm{KIM}^{2}$, Sukmo HONG ${ }^{2}$, \\ Seungkook $\mathrm{KO}^{1}$, Woon-kab $\mathrm{CHO}^{3}$ \\ ${ }^{1}$ Dept. of Physics, University Of Ulsan, Ulsan, 680-749, Korea \\ ${ }^{2}$ Pohamg Accelerator Laboratory, POSTECH, Pohang, 790-784, Korea \\ ${ }^{3}$ Korea Institute of Nuclear Safety, Daejeon, 305-338, Korea
}

\begin{abstract}
The accurate estimation of secondary particle production from thick targets by intermediate energy proton or heavy ion is very important factor to determine source term in radiation shielding design. Three well-proved Monte Carlo codes of the FLUKA, MCNPX, and PHITS were reviewed and benchmarking calculations for proton-induced neutrons and photons have been carried out. Neutron yields from Be, C, Al, and $\mathrm{Fe}$ target for 113 and $256 \mathrm{MeV}$ protons were calculated and compared with Meier's experimental data. From those comparisons, the property of above Monte Carlo codes could be observed for application of shielding design. The proton energies of $100,150,200$ and $230 \mathrm{MeV}$ were reviewed for thick $\mathrm{Al}, \mathrm{Fe}, \mathrm{Cu}$, and $\mathrm{Pb}$ targets to develop the source term. Dependence properties of neutron and photon production yields were found for target materials and target thickness. In this paper, a part of comparison results are presented and the discrepancies and agreements between each code are discussed for various target materials and proton energies.
\end{abstract}

KEYWORDS: Differential yield, FLUKA, MCNPX, PHITS, Proton, Intermediate energy

\section{Introduction}

In Korea, several intermediate energy proton and heavy ion accelerators have been considered for multiple applications including a cancer therapy or the construction of $100 \mathrm{MeV}$ protons linac is underway ${ }^{1)}$. Generally proton energy for a cancer therapy is 80 to $250 \mathrm{MeV}$ and carbon ion energy is up to $430 \mathrm{MeV} / \mathrm{u}$. The development of computational model composed of source term and attenuation length is very important in accelerator shielding calculation because of saving time and budget. On the other hand, Monte Carlo simulation codes have been used to give more precise shielding information for complicated structures or places where the detail data is requested.

Well-proved Monte Carlo codes like FLUKA ${ }^{2)}$, MCNPX ${ }^{3)}$, PHITS $^{4)}$, GEANT4 ${ }^{5)}$ and MARS ${ }^{6}$ are used for shielding calculation of high energy accelerator, but there are a little discrepancies between calculation results using each code at present ${ }^{7)}$ even though the same theoretical models are used. Therefore benchmarking calculation has been carried out using published well-known experimental data. In this study, the neutron and photon production yields calculated using above Monte Carlo codes were compared with Meier's measured results ${ }^{8,9}$. The production characteristics were observed in calculation results for various target materials and proton with energy range from $100 \mathrm{MeV}$ to $230 \mathrm{MeV}$. Target-dependent properties of production yields were investigated, which was necessary to determine shielding analysis models and to understand the codes. Finally optimum Monte Carlo code and calculation model for

*Corresponding Author, E-mail:lee@postech.ac.kr

(C) Atomic Energy Society of Japan

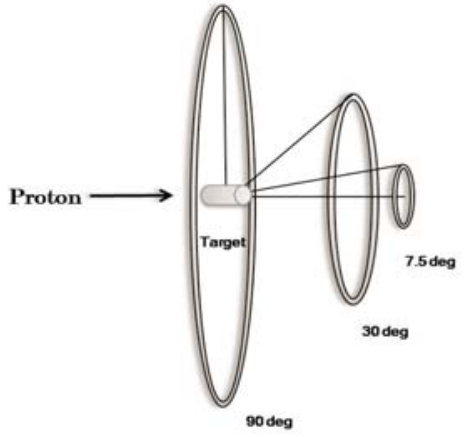

'ig. 1 Geometrical model of calculation of production yields using ICNPX and PHITS (cross-sectional shape of target is a circle or a sctangle)

shielding design are discussed.

\section{Methods and Simulation Model}

For the comparison and estimation of code performance, Meier's experimental data was used for a benchmarking data. When $113 \mathrm{MeV}^{8)}$ or $256 \mathrm{MeV}^{9}$ protons interacted with thick cylindrical targets of $\mathrm{Be}, \mathrm{C}, \mathrm{Al}$ and $\mathrm{Fe}$, the differential neutron yields were calculated using three Monte Carlo codes: FLUKA2008.3b, MCNPX2.6b, PHITS2.15. In FLUKA calculation, NEW-DEFA mode ${ }^{2)}$ was used. In PHITS calculation, the INC model, Bertini ${ }^{10)}$ and the Jaeri Quantum Molecular Dynamics (JQMD) ${ }^{11)}$ were employed with event generation mode for lower energy neutron. The mix and match method was employed in default calculation of MCNPX. The calculation using LA- $150^{12)}$ cross-section library were also compared. The geometrical mode of MCNPX and PHITS calculation was shown as Fig. 1. Target 
thicknesses in Table $\mathbf{1}$ are about proton range of that material. As done at Meier's experiments, differential yields were calculated at the angle of $7.530,60$, and 150 degrees to incident beam direction. The range of polar angle is $+/-1$ degree at each angle. A USRYIELD tally was used in FLUKA calculation and the boundary for above commend is assigned far from a target in order to neglect the uncertainty of angle due to thick target. F4 and track length tally are used in MCNPX and PHITS calculations, respectively. A parallel beam to moving direction with beam diameter of 0.5 $\mathrm{cm}$ was assumed in benchmarking calculations and its diameter of $0 \mathrm{~cm}$ was assumed in property study.

Table 1 Dimension of used targets in differential yield calculation for $113 \mathrm{MeV}$ protons

\begin{tabular}{lll}
\hline \hline Element & Radius $(\mathrm{cm})$ & Length $(\mathrm{cm})$ \\
\hline Beryllium & 3.65 & 5.70 \\
Carbon & 3.65 & 5.83 \\
Aluminum & 3.65 & 4.03 \\
Iron & 3.65 & 1.57 \\
\hline \hline
\end{tabular}

In the energy range which we have interested, the same style calculations were carried out in order to compare the property of each Monte Carlo code. The energies of incident protons are 100, 150, 200 and $230 \mathrm{MeV}$. Very thick rectangular targets of $\mathrm{Al}, \mathrm{Fe}, \mathrm{Cu}$, and $\mathrm{Pb}$ were used for this calculation. Four emission angles like 0 5, 45, 90 and 135 degrees at polar coordinate were chosen to get properties of angular distribution. At a forward angle, because the counting uncertainty was high, the forward angle range was assumed as 0 to 5 degrees.

One of goals of this study is to develop computational model of shielding calculation. Secondary particle production yields depending on target element shown in Table 2 and target thickness were evaluated using FLUKA code. The thickness dependency was obtained by the calculation for rectangular Fe targets of 1, 1.57, 8.8, and 17.6 cm thickness.

Table 2 Target thickness used in shielding calculation for 100 $230 \mathrm{MeV}$ protons

\begin{tabular}{lll}
\hline \hline Element & Target thickness $(\mathrm{cm})$ & Cross-section \\
\hline Aluminum & 18.00 & \\
Iron & 17.60 & $5 \mathrm{~cm} \mathrm{x} 5 \mathrm{~cm}$ \\
Copper & 14.50 & \\
Lead & 5.50 & \\
\hline \hline
\end{tabular}

\section{Results}

Monte Carlo calculations were carried out with low uncertainty less than $0.5 \%$ at most energy range. The benchmarking results for Meier's data ${ }^{8)}$ are shown in Fig. 2. The results calculated using FLUKA and PHITS agreed well with experimental results, but one using MCNPX didn't do.
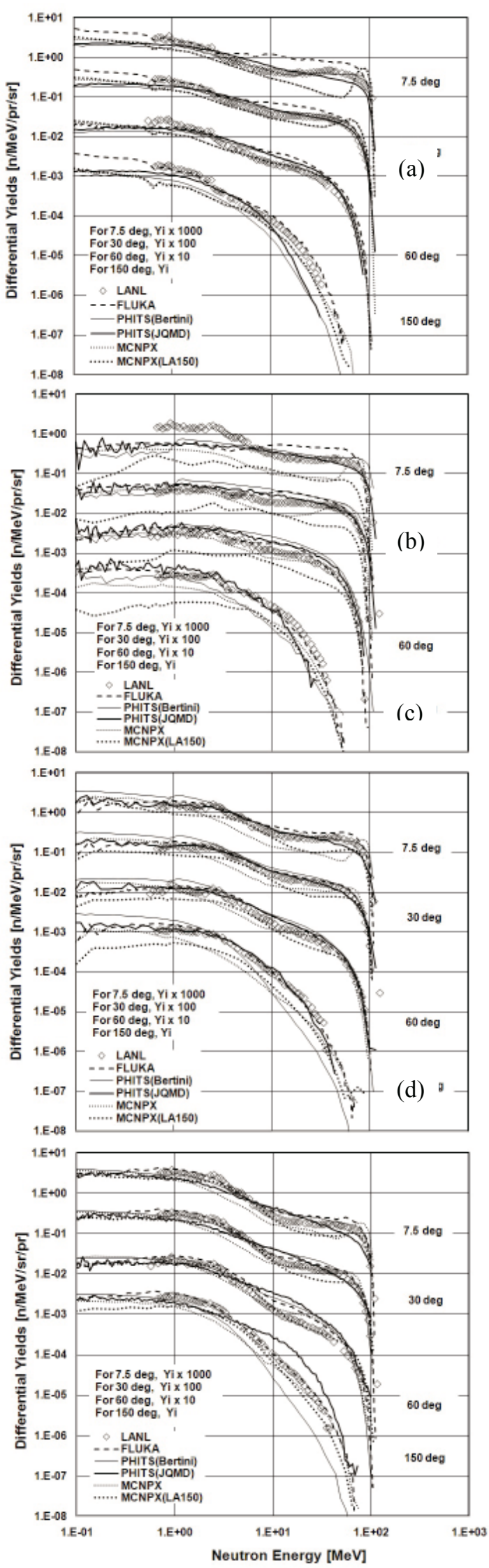

ig. 2 Comparison between calculated and measured differential eutron yield, Yi, from (a) Be, (b) C, (c) Al, and (d) Fe targets ombarded by $113 \mathrm{MeV}$ incident protons. LANL means Meier's xperimental results ${ }^{7)}$. 
Especially it showed strange hill-shape distribution. When the cross-section library, LA150, was used, it disappeared. It is also confirmed at calculated data of Meier's paper. But this is not shown at Tayama's paper ${ }^{13)}$ in which yields were described as lethargy unit. Lack of data library made large discrepancy as shown in cases of $\mathrm{C}$ and Al.

At 150 degrees, the results calculated using PHITS(Bertini) underestimated for every element. But the results have been improved when the JQMD model was used. The agreement between PHITS(JQMD) results and LANL experimental data was clearly better than one for PHITS(Bertini) results for all angles except 150 degrees in Fe target. Entirely PHITS calculation data well agreed with LANL data on higher energy range. FLUKA calculation showed good agreements at every energy range and at every angle.

Differential yields at every angle were found to have the same tendency each other independent of target element. Fig. 3 shows yields from thick Al target bombarded by $100 \mathrm{MeV}$ protons and $230 \mathrm{MeV}$ protons. The yields calculated using MCNPX increase continuously lower than $1 \mathrm{MeV}$. This tendency was also found at PHITS results. FLUKA results decrease at lower energy. In the comparison between

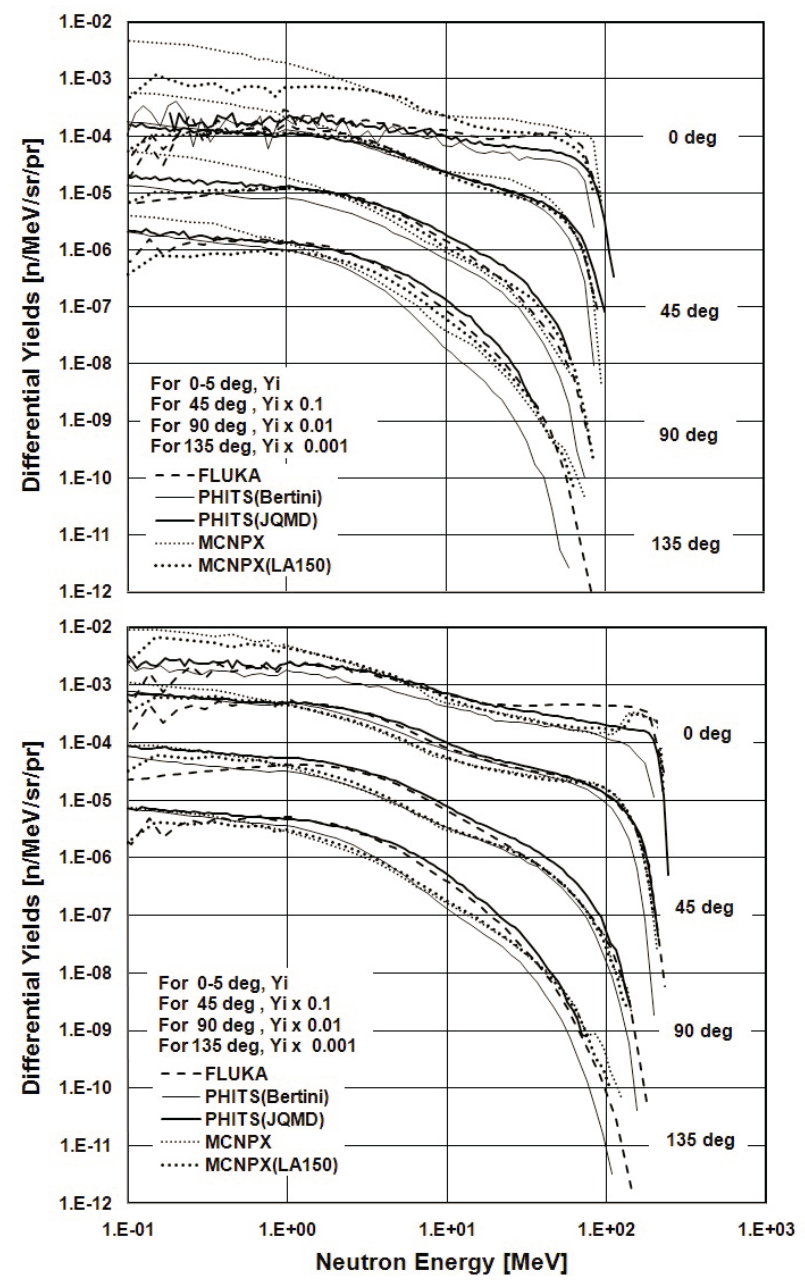

ig. 3 Comparison of differential neutron yields, Yi, from thick A rrget bombarded by $100 \mathrm{MeV}$ protons (Upper) and $230 \mathrm{Mel}$ rotons (Lower) between FLUKA, MCNPX, PHITS calculation.
FLUAK, MCNPX, and PHITS results, the discrepancy near 90 degrees was small relatively. The discrepancy increased a lot at forward or backward angles. It can be larger than factor of 5. In the case of the same target, the specific energydependent property was not found between $100 \mathrm{MeV}$ and $230 \mathrm{MeV}$. PHITS using MCNP code for low energy neutron transport showed the similar results with MCNPX, but FLUKA code gave small yields at lower energy than $1 \mathrm{MeV}$, relatively.

Higher differential yields were found at high $Z$ target as shown in Fig. 4 and the amount of increase is less than factor of 2 or 3 at higher neutron energy above $10 \mathrm{MeV}$. But below $10 \mathrm{MeV}$, the difference of yields between low $\mathrm{Z}$ and high $\mathrm{Z}$ target increased fast. The yields from $\mathrm{Pb}$ targets are 10 times higher than one from Al. That large difference was bigger than the difference due to different energies of incident proton. In Fig. 4, it is clear that the difference between bombardments of $100 \mathrm{MeV}$ proton and $230 \mathrm{MeV}$ smaller than one order of magnitude. That property is very important fact to be considered when someone determines shielding material.

Fig. 5 shows target thickness dependency of neutron yields and photon yields from Fe targets of various thicknesses. The tendency depending emission angle was found obviously. At 90 degrees, the neutron yields were almost the same to each other except of thinner target than proton-range. The difference increased dramatically at the forward angle of $0 \sim 5$ degrees, but maximum yields happened at thin target of proton-range like thickness. Therefore in developing computational model of shielding design, the most conservative idea is to use the yields from targets of proton-range like or thicker thickness as the source term. However at 135 degrees, thicker target generated larger neutron yields even though the difference between ones from different thickness targets was so small. A little different concept might be needed for backward direction. This tendency was also found at calculated photon yields.

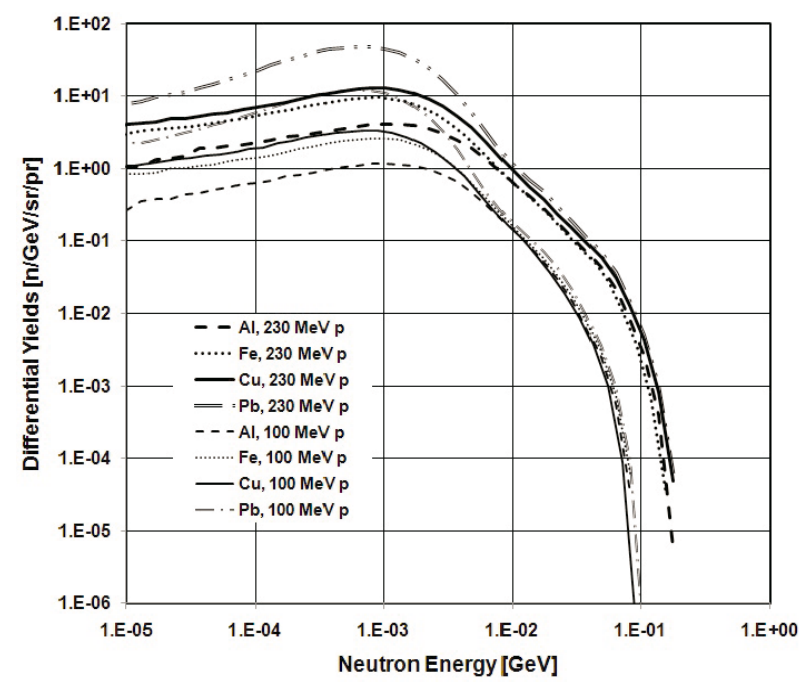

ig. 4 Target element dependency of differential neutron yields at 0 degrees from thick targets bombarded by $100 \mathrm{MeV}$ and 230 $\mathrm{teV}$ protons (in FLUKA calculation). 


\section{Conclusions}

In the shielding analysis, the source term can be evaluated through the calculation using Monte Carlo codes. The calculation results would give information of dose level directly where we have an interest in. But until now there is a little discrepancy between calculated data and experimental data and between calculated data using different Monte Carlo codes. The amount of its discrepancy was factor of two or three, which was equivalent to normal safety margin in shielding calculation

In this study, important facts were found in the view of shielding calculation. The neutron yields and photon yields are maximized at proton-range like thickness independent of elements of target materials.

Neutron yields increase proportional to the atomic number of target material. Especially $\mathrm{Pb}$ targets generate lots of neutrons below $10 \mathrm{MeV}$ relatively. The yield difference for different target element was larger than one for energy difference of incident proton at the range from $100 \mathrm{MeV}$ and $230 \mathrm{MeV}$. It should be considered at shielding analysis.

Authors also considered the benchmarking for Meier's experimental data using $256 \mathrm{MeV}$ protons and found the similar results to what is presented at this paper. $\mathrm{T}$. Nakamura's experimental data using $52 \mathrm{MeV}$ protons was also benchmarked. ${ }^{14)}$ As known generally, the calculation models and libraries are critical factors to determine consequential numbers like flux or dose equivalent. But it was found again at this study that the code-dependent results were not negligible even though the same model to each other was applied. More benchmarking studies and real experiment results are required continuously

H. Hirayama, et al.'s research ${ }^{7)}$ gave that there was important discrepancy between calculated results using every Monte Carlo code. It is found that such a tendency is still in the calculation of differential yields which determined.

\section{Acknowledgment}

This work has been supported by Nuclear Research and Development Fund of Korea Ministry of Education and Science.

\section{References}

1) B.H. Choi, et al., "STATUS OF THE PROTON ENGINEERING FRONTIER PROJECT", Proc. of PAC2005, Knoxville, Tennessee, 576 (2005).

2) A. Ferrari, et al., "FLUKA: a multi-particle transport code", CERN 2005-10, INFN/TC_05/11, SLAC-R-773, (2005).

3) D. B. Pelowitz, et al., "MCNPX User's Manual Version 2.6.0", LA-CP-07-1473, Los Alamos National Laboratory, (2007)

4) H. Iwase, et al., "Development of General-Purpose Particle and Heavy Ion Transport Monte Carlo Code", J. Nucl. Sci. Technol. 39 (11), 1142 (2002).

5) J. Allison, et al., "Geant4 developments and applications", IEEE Trans on Nuclear Science Vol. 53 No. 1, 270 (2006).

6) N. V. Mokhov, "The Mars Code System User's Guide", Fermilab-FN-628, Fermi National Laboratory, (1995).

7) H. Hirayama, et al., "Inter-comparison of medium-energy neutron attenuation in iron and concrete (7)", Proc. of SATIF-9,
Oak Ridge National Laboratory, (2008).
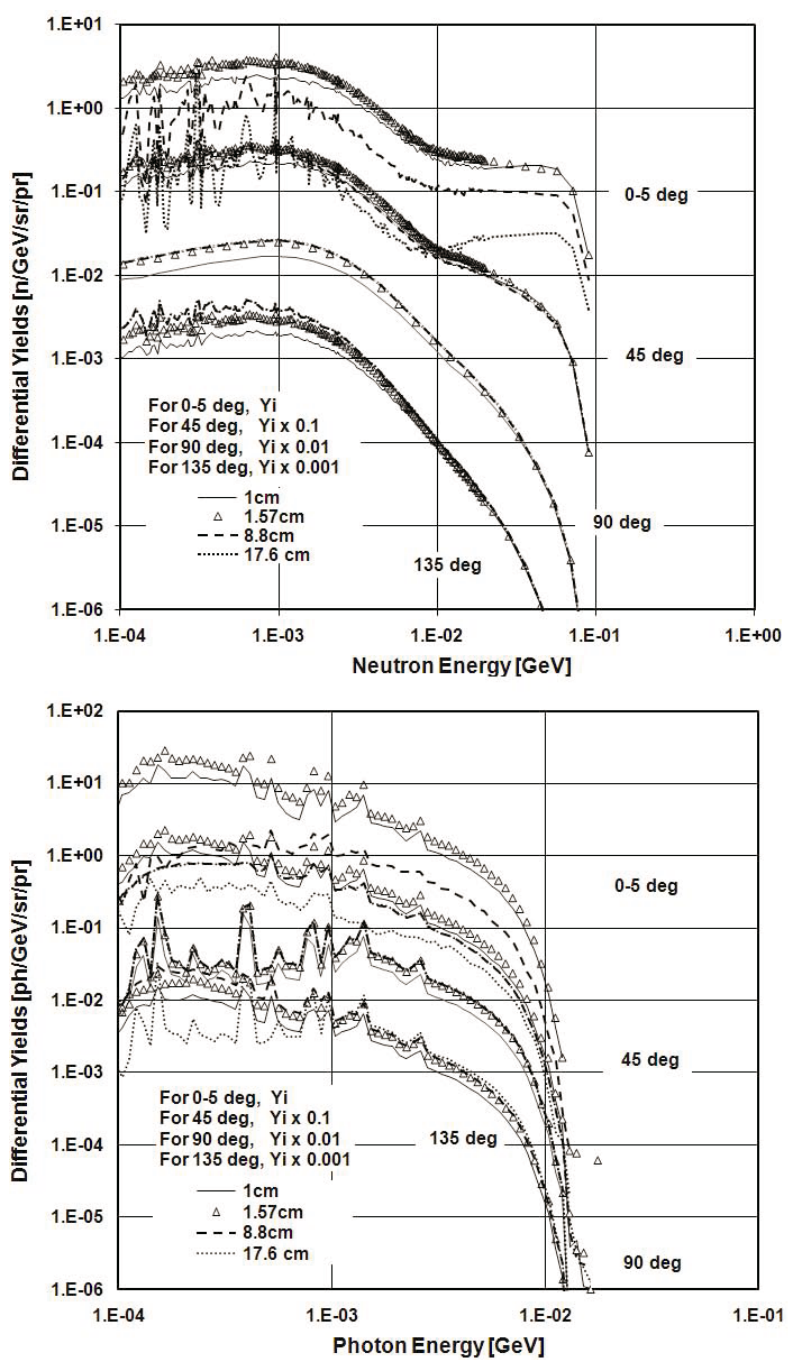

Fig. 5 Target thickness-dependency of neutron and photon yields from Fe target of various thickness bombarded by 100 $\mathrm{MeV}$ protons (in FLUKA calculation)

8) M. M. Meier, et al., "Differential neutron production cross sections and neutron yields from stopping-length targets for 113-MeV protons", Nucl. Sci. Eng. 102, 310 (1989).

9) M. M. Meier, et al., "Neutron Yields from Stopping -and Near-Stopping-Length Targets for $256 \mathrm{MeV}$ protons", Nucl. Sci. and Eng. 104, 339 (1990).

10) Bertini, H.W, "Low-Energy Intranuclear Cascade Calculation", Phys. Rev. 131 (1963).

11) Niita et al., "Analysis of the ( $\mathrm{N}, \mathrm{xN}^{\prime}$ ') reaction by quantum molecular dynamics plus statistical decay model", Phys Rev. C 52, 2620 (1995).

12) M. B. Chadwick, et al., "LA150 Documentation of Cross Sections, Heating, and Damage", LANL report; LA-UR-99-1222, Los Alamos National Laboratory (LANL) (1999).

13) R. Tayama, et al., "Benchmark calculations of neutron yields and dose equivalent from thick iron target for $52-256 \mathrm{MeV}$ protons “, Nucl. Eng. and Design 213, 119 (2002).

14) T. Nakamura, et al., "Neutron Production from Thick Targets of Carbon, Iron, Copper, and Lead by $30-$ and $52-\mathrm{MeV}$ Protons," Nucl. Sci. and Eng. 83, 444 (1983). 